\title{
The Bilinear method: a new stability-preserving order- reduction approach
}

\author{
T N Lucas*
}

Abstract

A new way of reducing the order of linear system transfer functions is presented. It guarantees stability in the approximation of stable systems and differs from existing stability-preserving methods by taking into account whole system parameter information when obtaining the approximate poles, not just that of the system poles. It uses a bilinear transformation in the process that renders the method more flexible than traditional techniques. Examples are given to highlight the advantages of the new approach.

Keywords: system order-reduction, stability preservation, bilinear mapping, Markov parameters.

* Division of Mathematical Sciences

University of Abertay Dundee

Bell Street

Dundee

DD1 1HG

Scotland, UK 


\section{INTRODUCTION}

For many years now, the use of reduced-order models in control system design has become a necessity, due to the ever-increasing complexity of the requirements to meet modern technological demands. In particular, the approach of linearising system, or sub-system, models before order reduction has proved very popular and successful (1-3).

Many sophisticated methods have evolved to reduce the order of linear systems (4) and each can usually boast its relative advantages over the others. Knowing which method to use has become something of an art among designers and of course depends upon the system characteristics to be approximated. Much of the information gathered when modelling a system tends to be in the frequency-domain (continuous or discrete) and thus the so-called frequency-domain order reduction methods retain a high profile in control system design.

These methods can be classified roughly into the two categories of:

(i) those that approximate or retain whole system parameters;

(ii) those that guarantee a stable reduced-order model from a stable system.

In category (i), the benchmark was set by, among others, the ideas of Chen and Shieh (5), Chuang (6) and Davidson and Lucas (7). Basically, these approaches matched as many system time moments (5) and Markov parameters (6) or general-point (shifted time moments) (7) as possible between the full and reduced-order models. In category (ii), the so-called Pole Retention (8), Routh (9), Schwarz (10), Stability Equation (11) and Differentiation (12) methods provided the foundation for others to build on.

It is generally recognised that approximations derived through the methods of category (i), when stable, tend to be better overall than those achieved by the methods of category (ii). To a large extent this is undoubtedly due to retaining more whole system information in the form of (shifted) time moments and/or Markov parameters. Although 
the methods of category (ii) can also retain whole system parameters, generally these amount to half the number preserved by those in category (i). The price to pay for guaranteed stability preservation is that the stable reduced-order transfer function's denominator is calculated first, from the full transfer function's denominator, leaving only its numerator to be obtained by matching the whole system parameters.

The purpose of this paper is to put forward a new stability-preserving orderreduction method for continuous-time transfer functions. It differs from existing methods in that, instead of using the full transfer function's denominator only to calculate the approximate poles, transformed whole system parameters are used to obtain these poles. It is seen that the system characteristics tend to be more accurately approximated using this approach than is the case for the more traditional stability-preserving methods. Further, a degree of flexibility is built into the method such that models can be improved upon by adjusting a single parameter in a bilinear transformation. Numerical examples are given to illustrate the advantages of the method.

\section{BASIS OF THE METHOD}

Lucas and Smith (13) recently developed a stability-preserving order reduction method for discrete-time transfer functions. They used the idea of matching whole system Markov parameters to the reduced-order model in a least-squares Padé approximation sense. The results obtained by this technique were quite startling and were seen to rival optimal models (14). It is therefore natural to ask whether this approach can be usefully employed in the continuous-time case and what are its advantages over existing stabilitypreserving methods? The answers to these questions will hopefully become clear in the following paragraphs. 
Consider the problem of reducing the stable, $n$ 'th order, linear, continuous-time transfer function, given in usual notation by

$$
G(s)=\frac{b_{n-1} s^{n-1}+\ldots . .+b_{1} s+b_{0}}{a_{n} s^{n}+\ldots \ldots . .+a_{1} s+a_{0}}
$$

to one of reduced order $k$, given by

$$
G_{k}(s)=\frac{d_{k-1} s^{k-1}+\ldots . .+d_{1} s+d_{0}}{s^{k}+e_{k-1} s^{k-1} \ldots \ldots . .+e_{1} s+e_{0}}
$$

Further, it is required that the poles of $G_{k}(s)$ are stable, that is, they all lie in the left-half of the s-plane. In order to satisfy this stability requirement, it is proposed to use a bilinear transformation on $G(s)$ such that its poles are mapped into the unit circle. This is achieved using the transformation

$$
s=\frac{2}{T}\left(\frac{z-1}{z+1}\right)
$$

which is a well-known transformation (15) used extensively in digital-filter design. It can be thought of as discretizing the continuous system by using the trapezium-rule approximation for the integral operators in the numerical solution of the governing system differential equation, where $T$ is the sampling time interval. For $T=2$, the standard bilinear mapping results and it will be shown that this often gives sufficiently accurate approximations. The computational effort required to carry out this transformation and its inverse is quite modest and can be achieved by an efficient Routh-type algorithm, as shown by Davies (16).

After applying this transformation, suppose that $G(s)$ is mapped to $H(z)$, given by

$$
H(z)=\frac{\beta_{n} z^{n}+\ldots \ldots+\beta_{1} z+\beta_{0}}{\alpha_{n} z^{n}+\ldots \ldots+\alpha_{1} z+\alpha_{0}}
$$


The whole system Markov parameters $m_{i}$ of $H(z)$ can now be calculated by a simple long-division algorithm, or by the recurrence relation

$$
\begin{aligned}
& m_{i}=\frac{1}{\alpha_{n}}\left\{\beta_{n-i}-\sum_{j=0}^{i-1} m_{j} \alpha_{n+j-i}\right\} \quad i=0,1,2, \ldots . \\
& \beta_{j}=0 \text { for } j<0
\end{aligned}
$$

where

$$
H(z)=\sum_{i=0}^{\infty} \frac{m_{i}}{z^{i}}
$$

The order reduction process begins with obtaining a reduced-degree transfer function denominator polynomial $D_{k}(z)$ in the z-domain approximation of $H(z)$. A stable approximation will be guaranteed (13) by finding the least-squares solution of the linear set

$$
\mathbf{M} \boldsymbol{\delta}=\boldsymbol{m}
$$

where, $\boldsymbol{\delta}=\left[\begin{array}{lllll}\delta_{k-1} & \delta_{k-2} & \ldots & \delta_{1} & \delta_{0}\end{array}\right]^{T}$ is the vector of the $k^{\prime}$ th degree denominator approximation $D_{k}(z)=z^{k}+\sum_{i=0}^{k-1} \delta_{i} z^{i}$

$$
\mathbf{M}=\left[\begin{array}{cccccc}
m_{k} & m_{k-1} & \cdot & \cdot & m_{2} & m_{1} \\
m_{k+1} & m_{k} & \cdot & \cdot & m_{3} & m_{2} \\
m_{k+2} & m_{k+1} & \cdot & \cdot & m_{4} & m_{3} \\
\cdot & \cdot & \cdot & \cdot & \cdot & \cdot \\
\cdot & \cdot & \cdot & \cdot & \cdot & \cdot \\
\cdot & \cdot & \cdot & \cdot & \cdot & \cdot
\end{array}\right] \quad \text { and } \quad \boldsymbol{m}=\left[\begin{array}{c}
-m_{k+1} \\
-m_{k+2} \\
-m_{k+3} \\
\cdot \\
\cdot \\
\cdot
\end{array}\right]
$$

Notice that equation (4) comes from directly equating the Markov parameters of $H(z)$ and its approximation $H_{k}(z)=N_{k}(z) / D_{k}(z)$; the structure of the resulting equations is such that the denominator approximation $D_{k}(z)$ can be found without reference to the numerator approximation $N_{k}(z)$. Further, this calculation involves (theoretically) all of 
the Markov parameters of $H(z)$ except $m_{0}$. Also, for a stable system, $\left|m_{i}\right| \rightarrow 0$ as $i \rightarrow \infty$, so that $\mathbf{M}$ and $\boldsymbol{m}$ can be truncated at a suitable point $(i>2 k)$.

The least-squares solution of equation (4) is given by

$$
\boldsymbol{\delta}=\left(\mathbf{M}^{T} \mathbf{M}\right)^{-1} \mathbf{M}^{T} \boldsymbol{m}
$$

which in turn specifies the pole locations in the z-domain of the reduced-order model. These poles will lie within the unit circle and thus applying the inverse bilinear transformation

$$
z=\frac{1+\frac{1}{2} s T}{1-\frac{1}{2} s T}
$$

to the approximate denominator polynomial will map its roots back into the left-half of the $s$-plane, giving the pole locations of the final reduced-order model.

It should be emphasised here that the main difference between the approximate transfer function's denominator obtained by this method and those of existing stabilitypreserving approaches is that of using whole system parameter information, albeit in a transformed domain. All of the Markov parameters (except the first) of $H(z)$ are used in the calculation, which approximate to the pulse response values of the discretization of $G(s)$. It is felt that matching these system parameters in a least-squares Padé sense enables an approximate pole distribution that is needed to reflect the system's transient response characteristics appropriately. Existing methods use the system pole distribution only and do not take the zero distribution into account when formulating these approximate poles. In the next section, example 1 illustrates the advantage of the suggested method over existing methods in this respect.

To calculate the numerator of the reduced-order transfer function it is proposed to use an existing sub-optimal technique (17). This minimises the integral-square-error (ISE) index, given the reduced-order transfer function's denominator, defined by 


$$
\int_{0}^{\infty}\left[y(t)-y_{k}(t)\right]^{2} d t
$$

where $y(t)$ and $y_{k}(t)$ are the responses of the full and approximate systems for a given input. Of course, the bilinear transformation of equations (3) and (6) can also be used to obtain the reduced-degree numerator polynomial, as can the more traditional methods of matching system time moments and/or Markov parameters in the $s$-domain. However, the ISE is a reliable measure of the accuracy of the approximation and is widely used in control system design. Also, because this algorithm is readily available and needs only the coefficients of the full system transfer function and those of the reduced-order transfer function's denominator, then it can be readily applied to obtain the optimal reduceddegree numerator for any existing stability-preserving method. This gives a fair basis on which to compare different techniques, allowing only the denominator derivations to be different.

In summary, the steps to follow to obtain the reduced-order transfer function by the Bilinear method are:

(i) apply the bilinear transformation $s=\frac{2}{T}\left(\frac{z-1}{z+1}\right)$ to $G(s)$ and obtain $H(z)$;

(ii) calculate sufficient Markov parameters of $H(z)$ to use in its approximation $H_{k}(z)$, resulting in equation (4);

(iii) obtain the stable approximate $z$-domain denominator polynomial $D_{k}(z)$ given by the least-squares solution in equation (5);

(iv) apply the inverse bilinear transformation $z=\frac{1+\frac{1}{2} s T}{1-\frac{1}{2} s T}$ to $D_{k}(z)$, mapping its roots into the left-half of the $s$-plane; 
(v) use the ISE minimisation algorithm to obtain the reduced-order transfer function's numerator for the given approximate denominator in step (iv).

\section{ILLUSTRATIVE EXAMPLES AND DISCUSSION OF RESULTS}

\section{Example 1}

To demonstrate in a simple way the advantage of using whole system parameters in deriving the approximate model's poles, consider a third-order system with poles at $-0 \cdot 5$ and $-1 \pm 2 \mathrm{j}$. The three cases of 0,1 and 2 system zeros will be considered respectively, as might be encountered in a typical design problem. The accuracy of the approximation is measured by the relative ISE value, defined by

$$
I=\int_{0}^{\infty}\left[y(t)-y_{k}(t)\right]^{2} d t \div \int_{0}^{\infty} y^{2}(t) d t
$$

where the full and approximate responses $y(t)$ and $y_{k}(t)$ respectively are due to an impulse input. In all three cases, the value of $T$ in equation (3) is taken to be 2 for initial simplicity, which corresponds to using the standard bilinear transformation. Also, the number of Markov parameters used in the least-squares Padé equation (4) is 30.

Although similar approximations result for half of this number, the stability preservation property (13) theoretically applies only when using the infinite number of Markov parameters of $H(z)$. So, taking a sufficiently large enough number ensures that this property will hold. Notice that when the moduli of the $m_{i}$ in equation (4) are very small, the corresponding equations do not make a significant contribution to the least-squares solution in equation (5) and so can be safely ignored.

(i) For no zeros, the system transfer function is given by

$$
G(s)=\frac{1}{2 s^{3}+5 s^{2}+12 s+5}
$$


Second-order models of the type given by equation (2), with $k=2$, are now calculated by the Bilinear method and the main stability-preserving methods, all enhanced by applying the algorithm to obtain optimal transfer function numerators after the denominator calculation (17). The results are shown in Table 1.

Table 1

\begin{tabular}{|l|cccc|c|}
\hline Method & $d_{1}$ & $d_{0}$ & $e_{1}$ & $e_{0}$ & $I$ \\
\hline Routh & -0.0409 & 0.2517 & 2.88 & 1.2 & $5.45 \%$ \\
Schwarz & -0.0278 & 0.1944 & 2.0 & 1.0 & $3.55 \%$ \\
Stability Equation & -0.0043 & 0.0664 & 0.4 & 1.0 & $44.69 \%$ \\
Differentiation & -0.0792 & 0.4661 & 4.8 & 3.0 & $18.02 \%$ \\
Bilinear & -0.0625 & 0.3456 & 3.6815 & 1.8851 & $8.63 \%$ \\
\hline
\end{tabular}

From these results, the Schwarz method gives the best ISE value followed closely by the Routh method, with the Bilinear method giving a reasonable thirdlowest value. Perhaps it is no surprise that, for systems with no zeros, the Schwarz and Routh methods should give such good approximations (although this also depends upon the pole distribution of the system) because the system characteristics are dominated by the information contained in the transfer function's denominator. However, the results for the Differentiation and Stability Equation methods are disappointing in this respect.

(ii) A minimum-phase zero at $s=-1$ is now introduced to the system, so that

$$
G(s)=\frac{s+1}{2 s^{3}+5 s^{2}+12 s+5}
$$

Reduction to a second-order model using the same methods as before gives the results in Table 2. 
Table 2

\begin{tabular}{|l|cccc|c|}
\hline Method & $d_{1}$ & $d_{0}$ & $e_{1}$ & $e_{0}$ & $I$ \\
\hline Routh & 0.0931 & 0.3008 & $2 \cdot 88$ & 1.2 & $27.05 \%$ \\
Schwarz & 0.1111 & 0.2222 & $2 \cdot 0$ & $1 \cdot 0$ & $22.84 \%$ \\
Stability Equation & 0.0603 & 0.0707 & 0.4 & 1.0 & $46.01 \%$ \\
Differentiation & 0.0065 & 0.7038 & 4.8 & 3.0 & $13.98 \%$ \\
Bilinear & -0.0289 & 0.8645 & 4.6768 & 4.27 & $6.00 \%$ \\
\hline
\end{tabular}

It is interesting to see from Table 2 that all of the existing stabilitypreserving methods give ISE values that are probably not acceptable. They also retain the same approximate poles as in the previous case, highlighting their insensitivity to the effect of the system zero. In contrast, the Bilinear method gives the best ISE value by far, with the poles being automatically adjusted through the matching of the whole system parameters.

(iii) Another (non minimum-phase) zero is now added to the system at $s=0.25$ to give

$$
G(s)=\frac{4 s^{2}+3 s-1}{2 s^{3}+5 s^{2}+12 s+5}
$$

As before, reducing this system to second-order models by the various methods gives the results in Table 3 .

Table 3

\begin{tabular}{|l|cccc|c|}
\hline Method & $d_{1}$ & $d_{0}$ & $e_{1}$ & $e_{0}$ & $I$ \\
\hline Routh & $2 \cdot 1820$ & -0.7474 & 2.88 & $1 \cdot 2$ & $20.41 \%$ \\
Schwarz & 1.6667 & -0.6667 & 2.0 & $1 \cdot 0$ & $29.34 \%$ \\
Stability Equation & 0.3190 & -0.3121 & 0.4 & 1.0 & $78.17 \%$ \\
Differentiation & 2.9342 & -0.7822 & 4.8 & 3.0 & $19.47 \%$ \\
Bilinear & 2.5849 & -0.2377 & 3.1388 & 4.3449 & $6.45 \%$ \\
\hline
\end{tabular}


Again, Table 3 shows starkly the effect that changing the system zeros has on the reduced-order models. Only the Bilinear method takes this information into account in the calculation of the approximate poles and gives probably the only acceptable reduced-order model.

\section{Example 2}

This example illustrates the flexibility of the given Bilinear method by changing the discretization parameter $T$ in equations (3) and (6). Consider the seventh-order system with transfer function

$$
G(s)=\frac{s^{5}+32 \cdot 5 s^{4}+380 s^{3}+2070 s^{2}+5424 s+2240}{s^{7}+15 s^{6}+124 s^{5}+630 s^{4}+2144 s^{3}+4600 s^{2}+5856 s+2880}
$$

which has poles at $-1,-2 \pm 2 \mathrm{j},-3 \pm 3 \mathrm{j},-2 \pm 4 \mathrm{j}$ and zeros at $-0 \cdot 5,-4 \pm 4 \mathrm{j},-10,-14$.

Third and second-order models are derived for this system by the Bilinear method, again using 30 Markov parameters of $H(z)$ in equation (4), for various values of $T$. For comparison, models derived by the Routh and Schwarz methods with optimal numerators (17) are also given in the form of equation (2) $(k=3$ and 2). The results are given in Tables 4 and 5 respectively.

Table 4

\begin{tabular}{|c|cccccc|c|}
\hline Method & $d_{2}$ & $d_{1}$ & $d_{0}$ & $e_{2}$ & $e_{1}$ & $e_{0}$ & $I$ \\
\hline Routh & -0.2106 & 6.6093 & 0.9123 & 3.4362 & 4.8629 & 2.3916 & $20 \cdot 88 \%$ \\
Schwarz & -0.1510 & 5.3914 & 2.5879 & 2.2175 & 5.7658 & 3.4164 & $8.73 \%$ \\
Bilinear $(T=2)$ & -1.0981 & 8.3064 & 5.7743 & 3.7883 & 9.7465 & 6.3748 & $7.65 \%$ \\
Bilinear $(T=1)$ & -0.9437 & 7.6026 & 4.3305 & 3.1630 & 8.6827 & 4.9796 & $5.14 \%$ \\
Bilinear $(T=0.5)$ & -0.4167 & 4.1373 & 17.4729 & 3.8967 & 11.2639 & 16.6711 & $3.92 \%$ \\
Bilinear $(T=0 \cdot 1)$ & -0.0303 & 0.8286 & 27.4918 & 3.6854 & 15.0923 & 23.0546 & $5.78 \%$ \\
\hline
\end{tabular}


Table 5

\begin{tabular}{|c|cccc|c|}
\hline Method & $d_{1}$ & $d_{0}$ & $e_{1}$ & $e_{0}$ & $I$ \\
\hline Routh & 1.4308 & 0.7882 & 1.6516 & 0.8123 & $57.59 \%$ \\
Schwarz & 1.2102 & 1.8446 & 1.9053 & 1.5407 & $51.98 \%$ \\
Bilinear $(T=2)$ & 0.6461 & 3.5013 & 1.3926 & 3.1818 & $23.60 \%$ \\
Bilinear $(T=1)$ & -0.2350 & 5.5405 & 1.4700 & 5.6121 & $6.38 \%$ \\
Bilinear $(T=0.5)$ & -0.6474 & 6.5467 & 1.7373 & 6.8504 & $4.28 \%$ \\
Bilinear $(T=0.1)$ & -0.1892 & 3.2447 & 0.6120 & 6.2355 & $29.83 \%$ \\
\hline
\end{tabular}

Of the third-order models in Table 4, only the Routh model gives a disappointing ISE value. There is little to choose between the Schwarz model and the (standard) Bilinear model for $T=2$; however, the ISE values decrease significantly in the Bilinear models for $T=1$ and $T=0 \cdot 5$. This ability to change the value of $T$ while still preserving stability in the model gives the Bilinear method an additional attribute that most existing methods do not have.

It is clear from the Bilinear model for $T=0 \cdot 1$ that the ISE value does not necessarily get smaller with $T$, so an element of judgement is required by the designer to decide on a satisfactory value for this parameter. Indeed, this could be an area for further research into the method. Further, it is interesting to note that the optimal third-order model for this system, i.e. the one that gives the actual minimum ISE value (18), has a relative ISE of $2 \cdot 12 \%$. The Bilinear model for $T=0 \cdot 5$ is seen to compare well with this value.

For the second-order models in Table 5, the rapid deterioration in the accuracy of the approximations is clear for most of the models; the exceptions being the Bilinear models for $T=1$ and $T=0.5$ respectively. The point made previously about making the value of $T$ too small is illustrated well in the case of $T=0 \cdot 1$, it looks to be a worse 
approximation than in the standard case of $T=2$. Again, it is of interest to note that the optimal second-order model for this system (18) gives a relative ISE value of $4 \cdot 13 \%$, which is close to that obtained by the Bilinear model for $T=0 \cdot 5$.

Figure 1 compares the impulse response curve of the full system with those of the third and second-order approximations obtained by the Bilinear method for $T=0 \cdot 5$. It is seen that both approximations are very good.

The method can also be used for step and polynomial inputs by considering the transient-response part of the system transfer function (19). For example, for a step input, the transfer function

$$
R(s)=\frac{G(s)-G(0)}{s}
$$

would be used by the method to obtain a reduced-degree denominator for the approximate poles. The reduced-degree numerator would then be obtained by ISE minimisation with the constraint that the first Markov parameter of $R(s)$ be retained. This will always be $-G(0)$ and ensures that a proper approximate transfer function $G_{k}(s)$ results from

$$
G_{k}(s)=s R_{k}(s)+G(0)
$$

Applying this procedure to the given seventh-order system, using $T=0 \cdot 5$, gives the thirdorder model

$$
G_{3}(s)=\frac{-1 \cdot 4298 s^{2}+8 \cdot 6854 s+4 \cdot 1718}{s^{3}+3 \cdot 2807 s^{2}+9 \cdot 1794 s+5 \cdot 3637}
$$

which has a relative ISE of $0.98 \%$, indicating an excellent approximation. This is confirmed in Figure 2, which shows a comparison of the step response curves for $G(s)$ and $G_{3}(s)$. 


\section{CONCLUDING REMARKS}

A new stability-preserving order-reduction method is presented that applies to continuous-time transfer functions. It differs from existing stability-preserving methods in that the approximate model's poles are calculated from whole system parameters rather than only from the system's poles.

The whole system parameters are the Markov parameters of the transformed transfer function, obtained through a bilinear mapping that essentially discretizes the continuous system. This also gives the method a degree of flexibility by being able to vary the sampling-time parameter $T$ in the bilinear transformation. The powerful leastsquares Padé method for discrete-time systems is then used to advantage to obtain the approximate stable pole distribution before mapping this back into the original $s$-plane. Finally, the method is enhanced by applying an existing ISE minimisation algorithm to calculate the reduced-degree numerator of the approximate transfer function.

Although the Bilinear method differs fundamentally from existing methods in the way that it obtains the approximate poles, the process remains two-staged. This means that the denominator and the numerator of the reduced-order transfer function are calculated successively, as in existing stability-preserving methods. As such, it is to be expected that sometimes reduced-order models obtained by methods that directly retain the maximum number of whole system parameters $(\mathbf{5 - 7 , 1 8})$ can give better overall approximations, when stable. Inevitably, this is part of the trade-off made by guaranteeing stability in the approximation. However, the examples given show that the method makes a valuable contribution to the stability-preserving approach in model order-reduction.

Overall, the Bilinear method has modest computational requirements, especially when compared to those having to utilise root-finding algorithms $(\mathbf{8}, \mathbf{1 1})$. The procedures 
of bilinear mapping, Markov parameter calculation, least-squares solution, inverse mapping and ISE minimisation all rely on efficient Routh-type algorithms and solving linear sets of equations. Perhaps, with today's available computing power, this aspect of a method is not quite so important as it once was. However, the Bilinear method's reliance only on well-established algorithms makes it attractive to use. 


\section{REFERENCES}

1 Owens, D. H. and Chotai, A. On the use of reduced order models and simulation data in control systems design. IMA J. Math. Control Inform., 1993, 10, 83 - 95.

2 Aguirre, L. A. Robust reference models for delayed systems. Proc. Instn. Mech. Engrs., Part I, J. of Systems and Control Engineering, 1994, 208 (I3), 197 - 199.

3 Green, M. and Limebeer, D. J. N. Linear Robust Control. Prentice-Hall Information Science Series, 1995 (Prentice-Hall, NJ).

4 Bultheel, A. and Van Barel, M. Padé techniques for model reduction in linear system theory: a survey. J. Comput. Appl. Math., 1986, 14, $401-438$.

5 Chen, C. F. and Shieh, L. S. A novel approach to linear model simplification. Int. J. Control, 1968, 8, $561-570$.

6 Chuang, S. C. Application of continued-fraction method for modelling transfer functions to give more accurate initial transient response. Electron. Lett., 1970, 6, $861-863$.

7 Davidson, A. M. and Lucas, T. N. Linear system reduction by continued-fraction expansion about a general point. Electron. Lett., 1974, 10, 271 - 273.

8 Shamash, Y. Linear system reduction using Padé approximation to allow retention of dominant modes. Int. J. Control, 1975, 21, 257 - 272.

9 Hutton, M. F. and Friedland, B. Routh approximations for reducing order of linear time-invariant systems. IEEE Trans. Autom. Control, 1975, 20, 329 - 337.

10 Lucas, T. N. and Davidson, A. M. Frequency-domain order reduction of linear systems by Schwarz approximation. Int. J. Control, 1983, 37, 1167 - 1178.

11 Chen, T. C., Chang, C. Y. and Han, K. W. Model reduction using the stability equation method and the continued-fraction method. Int. J. Control, 1980, 32, $81-94$. 
12 Gutman, P., Mannerfelt, C. F. and Molander, P. Contributions to the model reduction problem. IEEE Trans. Autom. Control, 1982, 27, $454-455$.

13 Lucas, T. N. and Smith, I. D. Discrete-time least-squares Padé order reduction: a stability-preserving method. Proc. Instn. Mech. Engrs., Part I, J. of Systems and Control Engineering, 1998, 212, 49 - 56.

14 Lucas, T. N. Optimal discrete model reduction by multipoint Padé approximation. J. Franklin Inst., 1993, 330, 855 - 867.

15 Sinha, N. K. Linear Systems, 1991 (John Wiley \& Sons, New York).

16 Davies, A. C. Bilinear transformation of polynomials. IEEE Trans. Circuits and Systems, 1974, 21, $792-794$.

17 Lucas, T. N. Sub-optimal model reduction by multipoint Padé approximation. Proc. Instn. Mech. Engrs., Part I, J. of Systems and Control Engineering, 1994, 208, $131-134$.

18 Lucas, T. N. Optimal model reduction by multipoint Padé approximation. $J$. Franklin Inst., 1993, 330, 79 - 93.

19 Lucas, T. N. Constrained optimal Padé model reduction. Trans. ASME, J. Dynam. Sys. Meas. and Control, 1997, 119, $685-690$. 


\section{Legends for Graphs}

Figure 1:

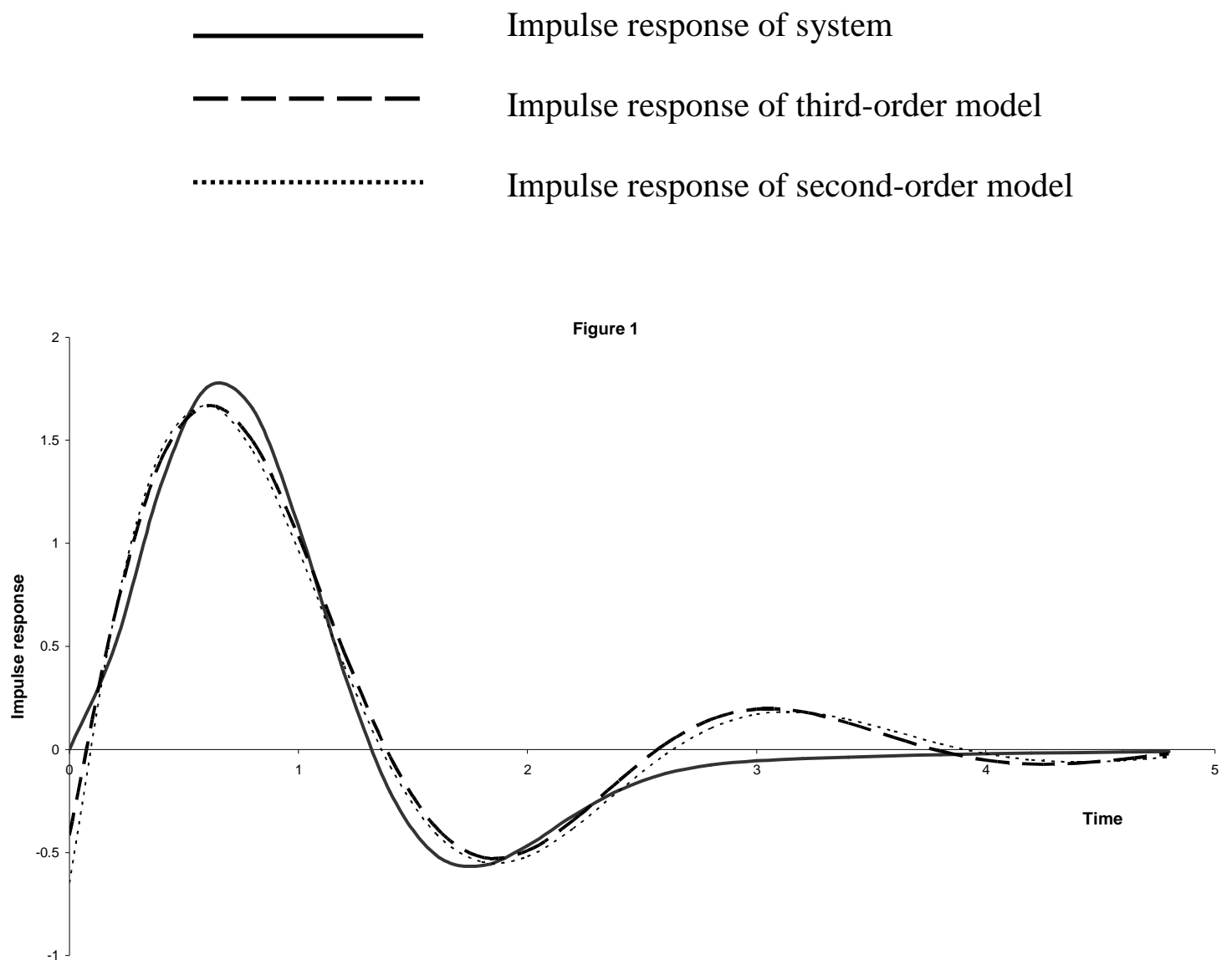

Figure 2:

Step response of system

- $-ー-ー \quad$ Step response of third-order model 


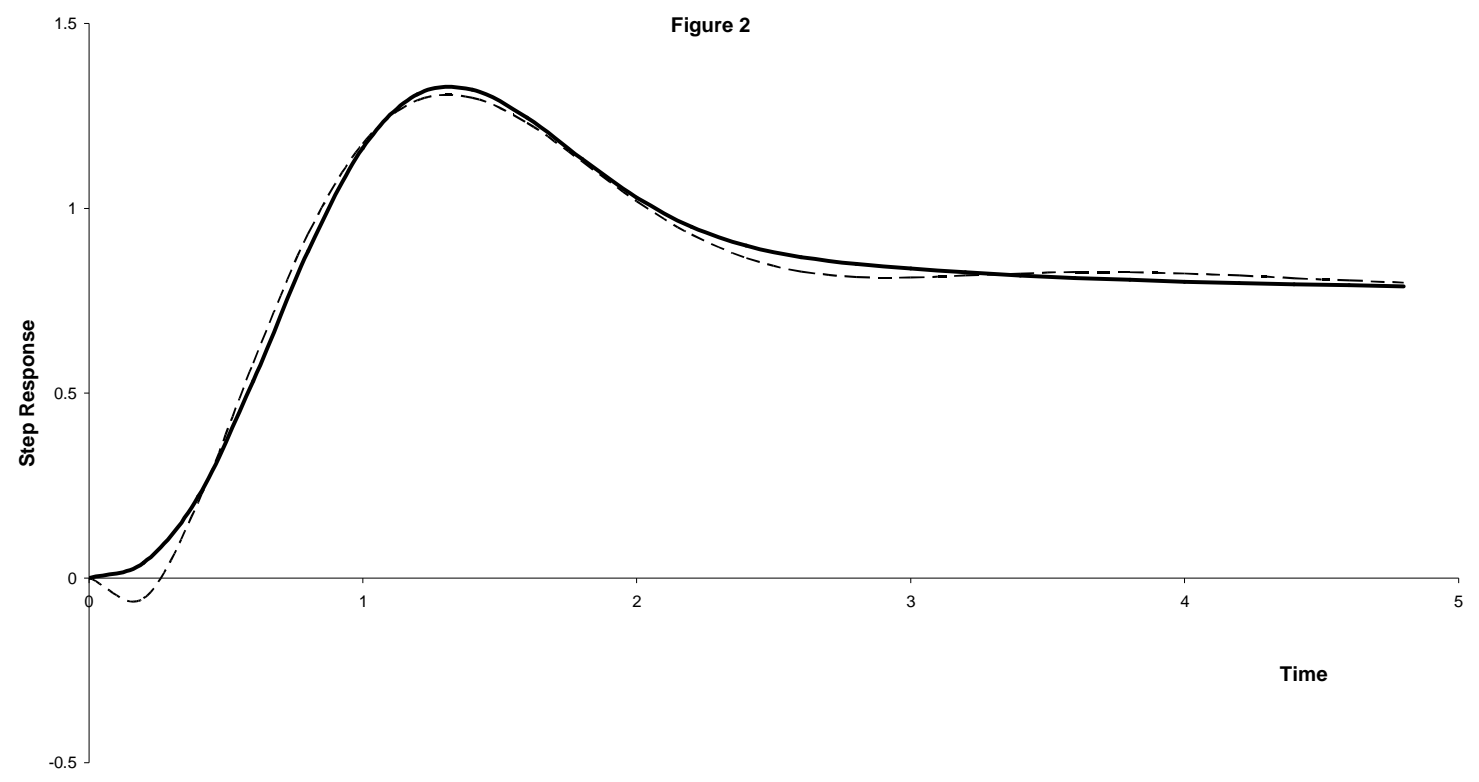

